\section{Perfil nutricional e fatores associados à ocorrência de desnutrição entre crianças indígenas Kaingáng da Terra Indígena de Mangueirinha, Paraná, Brasil}

\author{
Nutritional profile and factors associated with \\ malnutrition in Kaingáng children on the \\ Mangueirinha Indigenous Reserve, \\ Paraná State, Brazil
}

\author{
${ }_{1}^{1}$ Departamento de Nutrição, \\ Universidade Estadual do \\ Centro-Oeste, Guarapuava, \\ Brasil. \\ 2 Departamento de \\ Nutrição, Universidade \\ Federal de Santa Catarina, \\ Florianópolis, Brasil. \\ 3 Programa de Pós-graduação \\ em Epidemiologia, \\ Universidade Federal de \\ Pelotas, Pelotas, Brasil. \\ Correspondência \\ A. M. Kühl \\ Departamento de Nutrição, \\ Universidade Estadual do \\ Centro-Oeste. \\ C. P. 381, Guarapuava, $P R$ \\ 85100-970, Brasil. \\ adrimasiero@hotmail.com
}

\begin{abstract}
The goal of this study was to evaluate the nutritional status of indigenous children and to investigate factors associated with nutritional deficits. Weight and height measurements were obtained for 141 Kaingáng children from 0 to 5 years of age living on the Mangueirinha Indigenous Reserve in Paraná State, Brazil. Data on maternal and infant conditions and socioeconomic characteristics were also gathered through face-to-face interviews. Based on World Health Organization criteria (2006), 24.8\% of the children presented low height-for-age (HA), 9.2\% low weight-forage (WA), 2.1\% low weight-for-height (WH), and $2.1 \%$ low weight according to body mass index for age (BMIA). Based on NCHS criteria (1977), $19.9 \%$ of the children presented low HA, 9.2\% low WA, and $1.4 \%$ low WH. $6.4 \%$ were overweight according to BMIA. Low birth weight and non-masonry housing construction were associated statistically with nutritional deficits. The Kaingáng children are subject to poor living conditions, associated with deficits in their nutritional profile.
\end{abstract}

South American Indians; Nutritional Status; Anthropometry
Adriana Masiero Kühl 1

Arlete Catarina Tittoni Corso 2

Maurício Soares Leite 2

João Luiz Bastos 3

\section{Introdução}

Os povos indígenas constituem uma parcela da população brasileira ainda caracterizada pela escassez de informações epidemiológicas e demográficas, o que restringe o desenvolvimento de intervenções sociais e de saúde 1 . Visando à obtenção de informações demográficas, de morbidade, imunização, saúde bucal, nutrição, acompanhamento a gestantes, recursos humanos, infra-estrutura e saneamento, em 2000 foi criado o Sistema de Informações da Atenção em Saúde Indígena (SIASI) como um componente da Política Nacional de Atenção à Saúde dos Povos Indígenas. No entanto, apesar de suas potencialidades, o sistema ainda encontra-se apenas parcialmente implantado e enfrenta dificuldades que incluem os "instrumentos de coleta, [a] capacitação dos recursos humanos, [a] ausência de interface com os demais sistemas nacionais de informação em saúde, [a] dificuldade de acesso às informações e a não utilização das informações para o planejamento das ações" 2 (p. 858).

No que refere aos aspectos alimentares e nutricionais, a situação não chega a ser radicalmente distinta, na medida em que também prevalece uma relativa escassez de dados nesta área. Isso, a despeito do significativo aumento do número de publicações observado a partir da década de 1990 e da ampliação das faixas etárias e do número de etnias estudadas ${ }^{3}$. São poucos os estudos realizados na Região Sul do país com 
populações indígenas 4,5,6. Os dados disponíveis revelam um quadro em que predominam condições precárias de saneamento e um perfil de morbimortalidade caracterizado por freqüências elevadas de doenças infecciosas e parasitárias, registrando-se índices usualmente mais altos do que as médias regionais e nacionais 7,8 .

De modo geral, e apesar de uma considerável diversidade de perfis, os dados alimentares e nutricionais delineiam um quadro igualmente desfavorável. Entre adultos e adolescentes multiplicam-se os registros de sobrepeso e obesidade, além de desordens associadas, como dislipidemias, diabetes mellitus e hipertensão arterial 9,10,11,12,13. Entre crianças observam-se prevalências de moderadas a elevadas de baixa estatura e baixo peso para a idade, além de elevadas prevalências de anemia 14,15,16,17 e, em alguns casos, uma ampla inadequação das dietas 4,18,19. Em seu conjunto, esses dados apontam para condições amplamente desfavoráveis para a saúde e o crescimento infantil.

A realização desta pesquisa teve como objetivo a caracterização do perfil nutricional de crianças Kaingáng e a identificação dos fatores associados à ocorrência de déficits antropométricos nesta população.

\section{Métodos}

O presente estudo foi realizado na Terra Indígena de Mangueirinha, localizada no Município de Mangueirinha, sudoeste do Estado do Paraná, nas bacias dos rios Paraná e Iguaçu 20, totalizando uma área de 16.375 hectares. Nessa terra indígena vivem os povos Kaingáng e Guarani, sendo que os primeiros representam a população mais numerosa, totalizando 1.280 indivíduos, distribuídos em cinco localidades denominadas aldeia Sede ou Campina, Água Santa, Paiol Queimado, Fazenda ou Trevo e Mato Branco. Os centros urbanos mais próximos ficam a 10km (Mangueirinha) e $30 \mathrm{~km}$ (Coronel Vivida) da aldeia Sede; o acesso é feito por rodovias, havendo transporte urbano até a aldeia.

Os Kaingáng pertencem ao tronco lingüístico Macro-Jê e estão em contato permanente com não-índios desde o século XVIII. Atualmente, constituem um dos povos indígenas mais numerosos do país. Totalizando cerca de $25 \mathrm{mil}$ indivíduos distribuídos em mais de trinta terras indígenas nos estados de São Paulo, Paraná, Santa Catarina e Rio Grande do Sul 20, os Kaingáng encontram-se hoje confinados a uma pequena parcela de seus territórios originais. A Terra Indígena de Mangueirinha encontra-se cercada por fazendas e plantações de soja, e hoje pouco resta da cobertura vegetal nativa nas terras Kaingáng. O esgotamento dos recursos naturais é atualmente uma realidade, que acaba por comprometer as condições de alimentação e nutrição desse povo.

Com uma economia tradicionalmente baseada na agricultura, na caça e na colheita, os Kaingáng vivem hoje basicamente da agricultura, que produz alimentos como o milho, a abóbora e o feijão branco, da colheita de frutos como o pinhão, da venda de artesanato e de trabalho remunerado, geralmente em fazendas e em funções como as de professores indígenas e agentes indígenas de saúde. As aposentadorias também constituem uma fonte importante de renda para os Kaingáng. A agricultura é direcionada tanto para o consumo próprio como para a venda, mas, frente à restrição territorial e ao esgotamento dos recursos naturais, o acesso aos alimentos acontece também por meio da aquisição comercial, feita em grande parte nos mercados das cidades mais próximas e, eventualmente, pelo recebimento de cestas de alimentos. A dieta passou a ser basicamente amilácea e rica em gorduras, destacando-se o consumo de farinhas e carnes com maior quantidade de gorduras 4,21 .

Os Kaingáng também enfrentam sérios problemas de assistência à saúde, o que se reflete no perfil epidemiológico da população. Segundo levantamentos realizados em algumas comunidades indígenas Kaingáng, as principais causas de internação são as doenças respiratórias e as infecto-parasitárias 22,23 , sendo as principais causas de morte as doenças evitáveis por intermédio de atenção primária à saúde, como as respiratórias e a desnutrição 22. As doenças parasitárias atingem principalmente as crianças, como acontece na comunidade indígena de Rio das Cobras (Paraná), onde, segundo levantamento, 95\% das crianças avaliadas apresentavam algum tipo de parasita, principalmente Ascaris lumbricoides, seguido por Entamoeba coli 7 .

Dentre os fatores responsáveis por essa situação estão as precárias condições ambientais e sanitárias às quais os Kaingáng estão, de modo geral, expostos, como a falta de tratamento da água, a utilização de material reaproveitado para a construção de casas, a alta densidade familiar, a falta de energia elétrica, a ausência de saneamento básico, a contaminação do solo por dejetos depositados a céu aberto e a falta de coleta de lixo 4,6,7.

No presente estudo, foram incluídas todas as crianças Kaingáng menores de cinco anos de idade, de ambos os sexos, filhas de mãe e/ou pai pertencentes à etnia Kaingáng, residentes nas cinco aldeias durante o desenvolvimento do trabalho. Segundo os registros da unidade local 
de saúde, no período de realização da coleta de dados viviam ali 147 menores de cinco anos de idade. Entretanto, durante o trabalho de campo foram avaliadas 141 crianças, pois ocorreram 6 perdas (4\%) pelos seguintes motivos: (1) uma mãe não aceitou participar da pesquisa; (2) após três visitas à residência, as mães de cinco crianças não foram localizadas.

Para a avaliação do estado nutricional foram tomadas medidas antropométricas de peso e altura/comprimento e a data de nascimento de cada criança. Para a realização da antropometria foram utilizados os instrumentos e as técnicas recomendados pela Organização Mundial da Saúde (OMS) 24 para crianças até cinco anos de idade. A coleta dos dados antropométricos foi feita na unidade local de saúde das comunidades Sede e Trevo, durante dias de vacinação, por um único examinador (A.M.K.).

A classificação do estado nutricional foi realizada de acordo com os critérios das novas curvas adotadas pela OMS 25, por meio dos índices de altura para a idade (AI), peso para a idade (PI), peso para a altura (PA) e índice de massa corporal para a idade (IMCI), sendo classificadas como desnutridas aquelas crianças com escores $\mathrm{z}<-2 \mathrm{DP}$ e com sobrepeso as crianças com escores $z>2 D P$. Para garantir a comparabilidade com estudos anteriores, o estado nutricional infantil foi também descrito com base nos parâmetros recomendados pelo National Center for Health Statistics (NCHS) dos Estados Unidos 26, usando-se os índices de AI, PI e PA, utilizando os mesmos pontos de corte descritos anteriormente. Para ambas as populações-referência, a conversão das medidas das crianças kaingáng em escores $\mathrm{Z}$ foi feita através do módulo antropométrico WHO-Anthro-2005 (World Health Organization; http://www.who. int/childgrowth/software/en/) e do programa Epi Info versão 3.4.4 (Centers for Disease Control and Prevention, Atlanta, Estados Unidos).

As informações referentes às variáveis sócioeconômicas, maternas e das crianças foram obtidas usando-se um questionário padronizado. Esse questionário foi adaptado 27 e testado por meio de um estudo-piloto, realizado na comunidade Sede, com mães que não participaram do estudo principal. As seguintes variáveis foram verificadas:

a) Maternas: idade, escolaridade e estado civil;

b) Relativas às condições do domicílio: quantidade de cômodos, material das paredes, esgotamento sanitário e luz elétrica;

c) Da criança: peso ao nascer, diarréia nos últimos 15 dias, internação por pneumonia, sexo e idade.

A utilização dessas variáveis teve como base estudos anteriores 6,18,28 realizados em outras comunidades indígenas a fim de aprofundar o conhecimento a respeito das modificações ocorridas nas condições de vida desses povos e seus reflexos na saúde infantil. As informações a respeito da data de nascimento e do peso ao nascer foram obtidas no Cartão da Criança e as demais variáveis junto às mães.

As variáveis dependentes utilizadas na análise de associação foram o déficit de AI e o déficit de PI, considerando a classificação do estado nutricional de acordo com as recomendações da OMS 25. As análises foram realizadas no programa Stata 8 (Stata Corp., College Station, Estados Unidos), com estimação de razões de prevalência (RP) e respectivos intervalos de 95\% de confiança por meio da regressão de Poisson. A significância estatística das associações foi verificada com os testes de Wald para tendência linear e heterogeneidade. Consideraram-se significativos os resultados cujos valores de $\mathrm{p}$ bicaudais foram menores do que $5 \%$.

A inclusão das variáveis independentes nos modelos de regressão foi realizada por blocos. Inicialmente, foram inseridas as variáveis sócioeconômicas e demográficas da mãe. Em seguida, incluíram-se as variáveis relacionadas com as condições do domicílio e, finalmente, aquelas que dizem respeito à saúde da criança. Em cada bloco, a seleção das variáveis seguiu o procedimento de "eliminação para trás" (backward elimination), uma vez que todas as variáveis coletadas foram consideradas conceitualmente relevantes. Permaneceram nos modelos as variáveis cujos valores de $\mathrm{p}$ foram menores ou iguais a 0,20 . O ajuste para confundimento foi realizado para variáveis do mesmo bloco ou de blocos inseridos previamente nos modelos.

Tendo em vista que algumas crianças investigadas pertenciam a uma mesma família, o pressuposto de independência das observações foi violado. Assim, utilizou-se a opção survey psu do Stata, tomando-se as famílias como clusters em todas as análises.

A pesquisa obteve pareceres positivos para seu desenvolvimento pelo Comitê de Ética em Pesquisa com Seres Humanos (CEP-UFSC, protocolo no. 005/2006), Comissão Nacional de Ética em Pesquisa (CONEP, protocolo no. 13310) e Conselho Nacional de Desenvolvimento Científico e Tecnológico (CNPq), obtendo-se Autorização para Entrada em Terra Indígena da Fundação Nacional do Índio (FUNAI, protocolo no. 117/CGEP/06). As mães ou responsáveis pelas crianças participantes do estudo foram informadas sobre o conteúdo da pesquisa, bem como dos objetivos e finalidades da mesma por meio do Termo de Consentimento Livre e Esclarecido. 


\section{Resultados}

Das 141 crianças que participaram da pesquisa, $69(48,9 \%)$ eram do sexo feminino e $72(51,1 \%)$ do masculino (Tabela 1). O peso ao nascer das crianças apresentou uma distribuição próxima da normal, com média e mediana em torno de 3.000 g (desvio-padrão $-\mathrm{DP}=669$ ). A proporção de crianças nascidas com peso inferior a $2.500 \mathrm{~g}$ foi elevada (18,4\% - Tabela 1).

Em relação às características maternas, cerca de $14 \%$ das mães tinham menos de 20 anos, sendo que a média de idade foi de 27,1 anos $(\mathrm{DP}=7,9)$ e a mediana de 25 anos, caracterizando uma amostra de mães jovens. A baixa escolaridade predominou, já que $71,6 \%$ possuíam quatro anos de estudo ou menos. A maioria vivia em união estável (73,8\%) (Tabela 1).

No que se refere às condições de moradia, mais da metade $(63,1 \%)$ usa fossa séptica para os dejetos sanitários. Cerca de $21 \%$ dos domicílios apresentam material não-durável para construção das paredes, como madeira reaproveitada e lona. Mais de $80 \%$ deles possuem luz elétrica. A maioria das casas $(89,4 \%)$ é composta por dois ou três cômodos (Tabela 1).

A classificação do estado nutricional, usando-se os critérios recentemente propostos pela OMS 25, registrou uma elevada prevalência de déficit de AI, com uma em cada quatro crianças $(24,8 \%)$ apresentando este diagnóstico. O déficit de PI foi diagnosticado em $9,2 \%$ das crianças avaliadas. Tanto o índice peso para altura como o IMCI diagnosticaram apenas três crianças $(2,1 \%)$ como desnutridas (Tabela 2).

A adoção das curvas de referência propostas pelo NCHS 26 resultou em prevalências iguais a 19,9\% de déficit de AI, 9,2\% de PI e 1,4\% de PA (Tabela 2). Nenhuma das crianças apresentou indicadores compatíveis com a classificação de obesidade. No entanto, o sobrepeso atingiu 2,8\%, $3,6 \%$ e $6,4 \%$ delas segundo os índices PI, PA e IMCI, respectivamente, segundo a OMS 25, e 2,8\% de sobrepeso segundo o índice PI e 4,3\% segundo o índice PA conforme o NCHS 26.

Não houve diferenças estatisticamente significativas nas prevalências dos indicadores de PI e AI conforme o sexo da criança (Tabelas 3 e 4), embora os déficits fossem mais comuns entre as meninas (Tabelas 1, 3 e 4). A idade também não se mostrou significativamente associada, mas a mais alta proporção de desnutridos foi observada entre os mais jovens (Tabelas $1,3$ e 4$)$.

Nenhuma das variáveis de morbidade investigadas teve qualquer efeito significativo na prevalência do déficit de AI e de baixo PI. Da mesma forma que as variáveis acima referidas, nenhuma das características maternas esteve associada com o estado nutricional.

Crianças que nasceram com baixo peso apresentaram três vezes mais déficit de AI do que as que nasceram com peso igual ou superior a $2.500 \mathrm{~g}$ (Tabela 3). Na relação entre o baixo PI e o baixo peso ao nascer, as crianças com baixo peso ao nascer tiveram seis vezes mais déficit de PI do que aquelas que nasceram com peso adequado (Tabela 4).

Entre as condições de moradia investigadas, observou-se que crianças que moravam em habitações sem luz elétrica apresentaram o dobro do déficit de AI (43,5\%) em comparação com aquelas que moravam em casas com luz elétrica $(21,2 \%)$, apesar de esta diferença não ter sido estatisticamente significativa. No que se refere à prevalência de baixo PI, crianças que habitavam construções com paredes feitas de materiais não-duráveis, como lona e madeira reaproveitada, tiveram, significativamente, cerca de três vezes mais baixo PI do que as demais (Tabela 4).

\section{Discussão}

Em termos gerais, a antropometria Kaingáng revela um quadro semelhante àquele descrito para outras comunidades indígenas no país: manutenção da proporcionalidade corporal, revelada pelas baixas prevalências de baixo peso diagnosticadas pelos índices de PA e IMCI, e a baixa estatura apresentando-se como o principal déficit antropométrico, seguido pelo baixo PI 3,29. Vale assinalar que, a despeito da considerável magnitude dos déficits de estatura e de PI, a situação das crianças Kaingáng da Terra Indígena Mangueirinha situa-se num nível apenas intermediário de comprometimento, quando considerados os inquéritos antropométricos já realizados entre povos indígenas no Brasil.

A título de exemplo, e considerando-se a mesma faixa etária, as prevalências de baixa estatura já registradas entre outros povos indígenas ficam entre $10 \%$ e $61,7 \%$, enquanto as de baixo PI vão de $5 \%$ a mais de $50 \%{ }^{3}$. Ainda assim, a situação Kaingáng distancia-se enormemente das médias nacionais, nas quais se verifica $10,5 \%$ de baixa estatura 30 e 5,8\% de baixo PI ${ }^{31}$, e da Região Sul, onde se observa $5,1 \%$ de baixa estatura 30 e $2 \%$ de baixo PI 32 . Quando comparadas com outras crianças Kaingáng, os resultados encontrados demonstram um quadro mais favorável, pois prevalências de até $34,7 \%$ de baixa estatura e $12,9 \%$ de baixo peso já foram identificadas 6 .

Ao lado dos déficits antropométricos, contudo, a antropometria Kaingáng revela, por meio 
Distribuição absoluta e relativa da amostra conforme as categorias das variáveis independentes investigadas e prevalência de baixa altura para idade e baixo peso para idade em crianças Kaingáng entre 0 e 5 anos de idade. Terra Indígena de Mangueirinha, Paraná, Brasil, 2007.

\begin{tabular}{|c|c|c|c|c|}
\hline \multirow[t]{2}{*}{ Variáveis } & \multicolumn{2}{|c|}{$\begin{array}{l}\text { Distribuição da } \\
\text { amostra }\end{array}$} & \multirow{2}{*}{$\begin{array}{c}\text { Baixa altura } \\
\text { para idade } \\
\text { Prevalência (\%) }\end{array}$} & \multirow{2}{*}{$\begin{array}{c}\text { Baixo peso } \\
\text { para idade } \\
\text { Prevalência (\%) }\end{array}$} \\
\hline & $\mathrm{n}$ & $\%$ & & \\
\hline \multicolumn{5}{|l|}{ Escolaridade materna (anos) } \\
\hline$\leq 4$ & 101 & 71,6 & 25,7 & 11,9 \\
\hline$\geq 5$ & 40 & 28,4 & 22,5 & 2,5 \\
\hline \multicolumn{5}{|l|}{ Idade (anos) } \\
\hline $16-22$ & 47 & 33,3 & 23,4 & 8,5 \\
\hline $23-29$ & 47 & 33,3 & 27,7 & 4,3 \\
\hline $30-60$ & 47 & 33,3 & 23,4 & 14,9 \\
\hline \multicolumn{5}{|l|}{ Estado civil da mãe } \\
\hline União estável & 104 & 73,8 & 25,0 & 7,7 \\
\hline União instável/Separada/Solteira & 37 & 26,2 & 24,3 & 13,5 \\
\hline \multicolumn{5}{|l|}{ Luz elétrica no domicílio } \\
\hline $\operatorname{Sim}$ & 118 & 83,7 & 21,2 & 7,6 \\
\hline Não & 23 & 16,3 & 43,5 & 17,4 \\
\hline \multicolumn{5}{|l|}{ Número de cômodos no domicílio } \\
\hline 1 & 15 & 10,6 & 46,7 & 26,7 \\
\hline 2 & 96 & 68,1 & 24,0 & 6,3 \\
\hline 3 & 30 & 21,3 & 16,7 & 10,0 \\
\hline \multicolumn{5}{|l|}{ Material das paredes do domicílio } \\
\hline Durável & 112 & 79,4 & 23,2 & 6,3 \\
\hline Não-durável & 29 & 20,6 & 31,0 & 20,7 \\
\hline \multicolumn{5}{|l|}{ Esgotamento sanitário } \\
\hline Fossa séptica & 89 & 63,1 & 22,5 & 9,0 \\
\hline Fossa negra/Vala/Terreno/Mata & 52 & 36,9 & 28,9 & 9,6 \\
\hline \multicolumn{5}{|l|}{ Sexo da criança } \\
\hline Feminino & 69 & 48,9 & 29,0 & 10,1 \\
\hline Masculino & 72 & 51,1 & 20,8 & 8,3 \\
\hline \multicolumn{5}{|l|}{ Idade da criança (meses) } \\
\hline $0-18$ & 47 & 33,3 & 21,3 & 8,5 \\
\hline $19-40$ & 47 & 33,3 & 36,2 & 14,9 \\
\hline $41-60$ & 47 & 33,3 & 17,0 & 4,3 \\
\hline \multicolumn{5}{|l|}{ Diarréia nos últimos 15 dias } \\
\hline Não & 101 & 71,6 & 24,8 & 6,9 \\
\hline Sim & 40 & 28,4 & 25,0 & 15,0 \\
\hline \multicolumn{5}{|l|}{ Já foi internado por pneumonia? } \\
\hline Não & 104 & 73,8 & 26,9 & 11,5 \\
\hline Sim & 37 & 26,2 & 18,9 & 2,7 \\
\hline \multicolumn{5}{|l|}{ Baixo peso ao nascer } \\
\hline Não & 115 & 81,6 & 18,3 & 4,4 \\
\hline Sim & 26 & 18,4 & 53,9 & 30,8 \\
\hline Total & 141 & 100,0 & 24,8 & 9,2 \\
\hline
\end{tabular}

do IMCI 25, uma prevalência de 6,4\% de sobrepeso entre as crianças. Embora os registros de desnutrição infantil de modo geral caracterizem os perfis antropométricos infantis entre povos indígenas 3,29,33, não se trata de um achado isolado no contexto indígena. Estudos recentes vêm identificando casos de sobrepeso entre crianças de diversas etnias indígenas 6,17,18,34,35,36. No entanto, é entre os adultos que o sobrepeso e a obesidade assumem magnitudes elevadas e por vezes alarmantes 10,37,38. O surgimento de casos entre crianças e adolescentes 13,36,39, ainda que 
Distribuição absoluta e relativa de crianças Kaingáng menores de cinco anos de idade conforme o estado nutricional, segundo a classificação da OMS (2006) e do NCHS (1977). Terra Indígena de Mangueirinha, Paraná, Brasil, 2007.

\begin{tabular}{|c|c|c|c|c|}
\hline \multirow[t]{2}{*}{ Estado nutricional } & \multicolumn{2}{|c|}{ OMS (2006) } & \multicolumn{2}{|c|}{ NCHS (1977) } \\
\hline & $\mathbf{n}$ & $\%$ & $\mathbf{n}$ & $\%$ \\
\hline \multicolumn{5}{|c|}{ Baixa altura para idade } \\
\hline Sim & 35 & 24,8 & 28 & 19,9 \\
\hline Não & 106 & 75,2 & 113 & 80,1 \\
\hline \multicolumn{5}{|c|}{ Baixo peso para idade } \\
\hline Sim & 13 & 9,2 & 13 & 9,2 \\
\hline Não & 128 & 90,8 & 128 & 90,8 \\
\hline \multicolumn{5}{|c|}{ Baixo peso para altura } \\
\hline Sim & 3 & 2,1 & 2 & 1,4 \\
\hline Não & 138 & 97,9 & 139 & 98,6 \\
\hline \multicolumn{5}{|c|}{ Baixo índice de massa corporal para idade } \\
\hline Sim & 3 & 2,1 & - & - \\
\hline Não & 138 & 97,9 & - & - \\
\hline \multicolumn{5}{|c|}{ Sobrepeso segundo índice de altura para idade } \\
\hline Sim & 5 & 3,6 & - & - \\
\hline Não & 136 & 96,4 & - & - \\
\hline \multicolumn{5}{|c|}{ Sobrepeso segundo índice de massa corporal para idade } \\
\hline Sim & 9 & 6,4 & - & - \\
\hline Não & 132 & 93,6 & - & - \\
\hline Total & 141 & 100,0 & 141 & 100,0 \\
\hline
\end{tabular}

OMS: Organização Mundial da Saúde; NCHS: National Center for Health Statistics.

em prevalências reduzidas, ganha relevância não somente diante da magnitude do problema entre adultos em contextos específicos, mas também diante das evidências de importantes mudanças no estilo de vida dos povos indígenas. Essas geralmente acontecem em direção à redução dos níveis de atividade física e à ocidentalização da dieta, o que inclui o aumento do consumo de carboidratos simples e gorduras, e a redução da ingestão de fibras, e parecem estar associadas ao surgimento de doenças crônicas não-transmissíveis 9,11,37,40,41. Em seu conjunto, esses dados indicam estar em curso um processo de transição alimentar e nutricional entre os povos indígenas do país, com importantes repercussões para os perfis epidemiológicos e para os serviços que prestam atenção à saúde destes povos.

O peso ao nascer exerce influência decisiva no desenvolvimento pós-natal e afeta diretamente o crescimento físico, sendo o baixo peso ao nascer um importante fator de risco para o desenvolvimento de retardo no crescimento infantil, tornando as crianças mais suscetíveis à ocorrência de agravos de saúde ${ }^{42}$. Uchimura et al. 43 observaram entre crianças menores de um ano de idade da cidade de Maringá (Paraná) a maior ocorrência de déficit de AI entre aquelas que apresentaram baixo peso ao nascer, passando de $10,9 \%$ da população total para $52 \%$ entre as crianças com baixo peso ao nascer por retardo intra-uterino e $30 \%$ para as prematuras.

Entre as crianças Kaingáng, a prevalência de baixo peso ao nascer atingiu $18,4 \%$ das menores de cinco anos de idade, um percentual bastante elevado se comparado os resultados da Pesquisa Nacional sobre Demografia e Saúde 30, de 8,2\%, e com a prevalência observada na Região Sul do país, de 8,5\% para crianças não-indígenas 44 . Em se tratando de crianças indígenas em geral, os poucos estudos disponíveis apontam para elevadas freqüências de baixo peso ao nascer, com índices que chegam a atingir 30,4\% das crianças Kaiowá e Guaraní (Mato Grosso do Sul) 45, 17\% das Teréna da comunidade Córrego do Meio (Mato Grosso do Sul) 18 e 12\% das crianças Teréna de Buriti (Mato Grosso do Sul) 28.

No que se refere aos fatores associados ao estado nutricional, como esperado o baixo peso ao nascer teve influência negativa no crescimento estatural e ponderal das crianças. Após o nascimento, os déficits antropométricos estive- 
Razões de prevalências (RP) brutas e ajustadas e respectivos intervalos de 95\% de confiança (IC95\%) para a associação das variáveis maternas, do domicílio e da criança com deficit de altura para idade em crianças Kaingáng menores de cinco anos. Terra Indígena de Mangueirinha, Paraná, Brasil, 2007.

\begin{tabular}{|c|c|c|c|c|}
\hline \multirow[t]{2}{*}{ Variáveis } & \multicolumn{2}{|c|}{ Análise bruta } & \multicolumn{2}{|c|}{ Análise ajustada } \\
\hline & RP (IC95\%) & Valor de $\mathrm{p}$ & $\mathrm{RP}(\mathrm{IC} 95 \%)$ * & Valor de $\mathrm{p}$ \\
\hline Escolaridade materna (anos) & & 0,718 * & & 0,718 * \\
\hline$\leq 4$ & 1,0 & & 1,0 & \\
\hline$\geq 5$ & $0,9(0,4-1,8)$ & & $0,9(0,4-1,8)$ & \\
\hline Idade (anos) & & $1,000 * *$ & & 0,916 ** \\
\hline $16-22$ & 1,0 & & 1,0 & \\
\hline $23-29$ & $1,2(0,6-2,5)$ & & $1,2(0,5-2,5)$ & \\
\hline $30-60$ & $1,0(0,5-2,2)$ & & $1,0(0,5-2,1)$ & \\
\hline Estado civil da mãe & & 0,937 * & & 0,983 * \\
\hline União estável & 1,0 & & 1,0 & \\
\hline União instável/Separada/Solteira & $1,0(0,5-1,9)$ & & $1,0(0,5-2,0)$ & \\
\hline Luz elétrica no domicílio & & 0,033 * & & 0,169 * \\
\hline Sim & 1,0 & & 1,0 & \\
\hline Não & $2,1(1,1-4,0)$ & & $1,7(0,8-3,4)$ & \\
\hline Número de cômodos no domicílio & & $0,061 * *$ & & 0,186 ** \\
\hline 1 & 1,0 & & 1,0 & \\
\hline 2 & $0,5(0,2-1,1)$ & & $0,7(0,3-1,4)$ & \\
\hline 3 & $0,4(0,1-1,0)$ & & $0,5(0,2-1,4)$ & \\
\hline Material das paredes do domicílio & & 0,392 * & & 0,911 * \\
\hline Durável & 1,0 & & 1,0 & \\
\hline Não-durável & $1,3(0,7-2,6)$ & & $1,0(0,4-2,1)$ & \\
\hline Esgotamento sanitário & & 0,434 * & & 0,858 * \\
\hline Fossa séptica & 1,0 & & 1,0 & \\
\hline Fossa negra/Vala/Terreno/Mata & $1,3(0,7-2,4)$ & & $0,9(0,5-1,8)$ & \\
\hline Sexo da criança & & 0,273 * & & 0,175 * \\
\hline Feminino & 1,0 & & 1,0 & \\
\hline Masculino & $0,7(0,4-1,3)$ & & $0,7(0,4-1,2)$ & \\
\hline Idade da criança (meses) & & 0,618 ** & & 0,546 ** \\
\hline $0-18$ & 1,0 & & 1,0 & \\
\hline $19-40$ & $1,7(0,9-3,3)$ & & $1,7(0,9-3,3)$ & \\
\hline $41-60$ & $0,8(0,3-1,9)$ & & $0,8(0,3-1,7)$ & \\
\hline Diarréia nos últimos 15 dias & & 0,979 * & & 0,574 * \\
\hline Não & 1,0 & & 1,0 & \\
\hline $\operatorname{Sim}$ & $1,0(0,5-2,1)$ & & $0,8(0,4-1,6)$ & \\
\hline Já foi internado por pneumonia? & & 0,404 * & & 0,328 * \\
\hline Não & 1,0 & & 1,0 & \\
\hline Sim & $0,7(0,3-1,6)$ & & $0,7(0,3-1,5)$ & \\
\hline Baixo peso ao nascer & & 0,002 * & & $<0,001$ * \\
\hline Não & 1,0 & & 1,0 & \\
\hline Sim & $2,9(1,5-5,8)$ & & $3,0(1,7-5,3)$ & \\
\hline
\end{tabular}

* Teste de Wald para heterogeneidade;

** Teste de Wald para tendência linear.

ram associados com o tipo de material de construção das paredes do domicílio. Fatores que traduzem as condições de moradia, como luz elétrica e número de cômodos, têm sido consistentemente associados à ocorrência de agravos nutricionais $42,46,47$ e sua relação evidenciada por meio de estudos realizados com populações não-indígenas 48,49,50. Crianças que residiam em habitações com paredes feitas de materiais nãoduráveis como madeira reaproveitada e lona tiveram cerca de três vezes mais baixo PI do que as demais. 
Razões de prevalências (RP) brutas e ajustadas e respectivos intervalos de $95 \%$ de confiança (IC95\%) para a associação das variáveis maternas, do domicílio e da criança com déficit de peso para idade em crianças Kaingáng menores de cinco anos. Terra Indígena de Mangueirinha, Paraná, Brasil, 2007.

\begin{tabular}{|c|c|c|c|c|}
\hline \multirow[t]{2}{*}{ Variável } & \multicolumn{2}{|c|}{ Análise bruta } & \multicolumn{2}{|c|}{ Análise ajustada } \\
\hline & RP (IC95\%) & Valor de $p$ & $\mathrm{RP}(\mathrm{IC} 95 \%)$ * & Valor de $\mathrm{p}$ \\
\hline Escolaridade materna (anos) & & 0,126 * & & 0,126 * \\
\hline$\leq 4$ & 1,0 & & 1,0 & \\
\hline$\geq 5$ & $0,2(0,0-1,6)$ & & $0,2(0,0-1,6)$ & \\
\hline Idade (anos) & & 0,356 ** & & 0,443 ** \\
\hline $16-22$ & 1,0 & & 1,0 & \\
\hline $23-29$ & $0,5(0,1-2,6)$ & & $0,5(0,1-2,4)$ & \\
\hline $30-60$ & $1,8(0,5-5,7)$ & & $1,5(0,5-4,6)$ & \\
\hline Estado civil da mãe & & 0,328 * & & 0,240 * \\
\hline União estável & 1,0 & & 1,0 & \\
\hline União instável/Separada/Solteira & $1,8(0,6-5,5)$ & & $1,9(0,6-5,9)$ & \\
\hline Luz elétrica no domicílio & & 0,200 * & & 0,399 * \\
\hline $\operatorname{Sim}$ & 1,0 & & 1,0 & \\
\hline Não & $2,3(0,6-8,1)$ & & $1,6(0,6-4,4)$ & \\
\hline Número de cômodos no domicílio & & 0,373 ** & & 0,849 ** \\
\hline 1 & 1,0 & & 1,0 & \\
\hline 2 & $0,2(0,1-0,9)$ & & $0,6(0,1-2,6)$ & \\
\hline 3 & $0,4(0,1-1,7)$ & & $1,0(0,2-4,9)$ & \\
\hline Material das paredes do domicílio & & 0,030 * & & 0,045 * \\
\hline Durável & 1,0 & & 1,0 & \\
\hline Não-durável & $3,3(1,1-9,8)$ & & $2,9(1,0-8,2)$ & \\
\hline Esgotamento sanitário & & 0,910 * & & 0,374 * \\
\hline Fossa séptica & 1,0 & & 1,0 & \\
\hline Fossa negra/Vala/Terreno/Mata & $1,1(0,3-3,5)$ & & $0,6(0,2-1,8)$ & \\
\hline Sexo da criança & & 0,731 * & & 0,702 * \\
\hline Feminino & 1,0 & & 1,0 & \\
\hline Masculino & $0,8(0,3-2,5)$ & & $1,3(0,3-4,8)$ & \\
\hline Idade da criança (meses) & & $0,409 * *$ & & 0,587 ** \\
\hline $0-18$ & 1,0 & & 1,0 & \\
\hline $19-40$ & $1,8(0,5-5,6)$ & & $1,7(0,5-5,7)$ & \\
\hline $41-60$ & $0,5(0,1-2,7)$ & & $0,6(0,1-2,8)$ & \\
\hline Diarréia nos últimos 15 dias & & 0,164 * & & 0,408 * \\
\hline Não & 1,0 & & 1,0 & \\
\hline $\operatorname{Sim}$ & $2,2(0,7-6,5)$ & & $1,5(0,5-4,3)$ & \\
\hline Já foi internado por pneumonia? & & 0,163 * & & 0,074 * \\
\hline Não & 1,0 & & 1,0 & \\
\hline $\operatorname{Sim}$ & $0,2(0,0-1,8)$ & & $0,2(0,0-1,2)$ & \\
\hline Baixo peso ao nascer & & 0,001 * & & $<0,001$ * \\
\hline Não & 1,0 & & 1,0 & \\
\hline Sim & $7,1(2,3-22,0)$ & & $6,2(2,3-16,8)$ & \\
\hline
\end{tabular}

* Teste de Wald para heterogeneidade;

** Teste de Wald para tendência linear.

A associação entre déficits antropométricos infantis e condições de moradia concordam, em termos gerais, com aqueles observados em contextos não-indígenas 51,52. A despeito da especificidade dos contextos indígenas e do cuidado exigido pelo exame de associações verificadas em outras populações, esse achado faz sentido, no caso Kaingáng, diante do prolongado tempo de contato com não-índios e do grau de interação da população Kaingáng com a população regional não-indígena. Assim é que se entre outros povos indígenas o uso de materiais não-duráveis 
para a construção de casas não necessariamente reflete condições sócio-econômicas desfavoráveis, entre os Kaingáng a associação encontrada sugere que ele assume significado semelhante àquele observado entre não-indígenas e, em certa medida, traduz condições precárias de vida.

Dinâmica semelhante é encontrada entre outras populações indígenas com elevado tempo de contato com não-índios, como os Kaingáng de Guarita 6 e os Teréna 18. Entre os primeiros, evidenciou-se a influência do analfabetismo materno, da fonte de água de consumo doméstico, do destino dos dejetos e da ausência de geladeira na residência sobre o crescimento das crianças 6 , enquanto que entre os Teréna 28 assinalam a influência da escolaridade materna na determinação do estado nutricional infantil.

Um exame mais detalhado das categorias de peso ao nascer e das condições de habitação revela, nos grupos que indicam melhores condições, prevalências muito inferiores de déficits antropométricos (Tabela 1). No entanto, à exceção do peso ao nascer, a análise das demais variáveis revela a manutenção de diferenciais importantes entre as magnitudes dos déficits antropométricos observados entre índios e não-índios.

Chama atenção a influência do peso ao nascer: crianças que nasceram com mais de $2.500 \mathrm{~g}$ apresentaram uma prevalência de baixo PI de $4,4 \%$, equivalente àquela registrada entre não-ín- dios. No que se refere às condições de habitação, crianças que viviam em domicílios com materiais duráveis nas paredes apresentaram prevalências de baixo PI equivalente a 6,3\%, aproximando-se bastante, portanto, da média nacional $(5,8 \%) 31$, mas ainda distante da média regional $(2 \%) 32$. A prevalência de baixa estatura foi de $23,2 \%$ quando as paredes eram construídas com material durável, enquanto as médias nacional e regional eram equivalentes a $10,5 \%$ e $5,1 \% 30$.

A situação das crianças Kaingáng de Mangueirinha aponta para uma realidade precária, na qual os indicadores do estado nutricional refletem condições ambientais e sócio-econômicas amplamente desfavoráveis para o crescimento infantil. A despeito das especificidades sócio-culturais que modulam o processo saúde-doença entre os povos indígenas, os fatores associados aos déficits antropométricos assemelham-se aos observados em contextos não-indígenas. À luz da trajetória Kaingáng, esse dado pouco surpreende e revela como os termos historicamente desiguais dessa interação aproximam os Kaingáng das condições de vida dos segmentos menos favorecidos da sociedade brasileira. Mesmo essa proximidade, contudo, é limitada, na medida em que se mantêm importantes diferenciais entre os indicadores nutricionais registrados entre os Kaingáng e entre os não-índios, em especial na Região Sul.

\section{Resumo}

Este estudo objetivou avaliar o estado nutricional de crianças indigenas e verificar os fatores associados à ocorrência de agravos nutricionais. Foram avaliadas 141 crianças Kaingáng de 0 a 60 meses de idade da Terra Indígena de Mangueirinha, Paraná, Brasil, por meio das medidas de peso e altura ou comprimento. Realizaram-se entrevistas com a aplicação de um questionário sobre condições materno-infantis e sócioeconômicas. Segundo os parâmetros da Organização Mundial da Saúde (2006), 24,8\% das crianças apresentavam déficit de altura para idade (AI), 9,2\% baixo peso para idade (PI), 2,1\% baixo peso para altura (PA) e 2,1\% baixo peso segundo o índice de massa corpo- ral para idade (IMCI). De acordo com o NCHS (1977), 19,9\% das crianças apresentaram baixa AI, 9,2\% baixo PI e 1,4\% baixo PA. O sobrepeso chegou a 6,4\%, segundo o IMCI. O baixo peso ao nascer e os materiais utilizados na construção das paredes das casas estiveram estatisticamente associados com déficits antropométricos. Este estudo mostra que as crianças Kaingáng estão inseridas em um contexto marcado por precárias condições de vida, que estão associadas com indicadores desfavoráveis do estado nutricional.

Índios Sul-Americanos; Estado Nutricional; Antropometria 


\section{Colaboradores}

A. M. Kühl participou da elaboração do desenho do estudo, da coleta das informações, da montagem do banco de dados, da análise estatística e da elaboração do manuscrito. A. C. T. Corso participou da elaboração do desenho do estudo, da montagem do banco de dados, da análise estatística e da elaboração do manuscrito. M. S. Leite participou da elaboração do manuscrito. J. L. Bastos participou da análise estatística e da elaboração do manuscrito.

\section{Referências}

1. Santos RV, Coimbra Jr. CEA. Cenários e tendências da saúde e da epidemiologia dos povos indígenas do Brasil. In: Coimbra Jr. CEA, Santos RV, Escobar AL, organizadores. Epidemiologia e saúde dos povos indígenas no Brasil. Rio de Janeiro: Editora Fiocruz; 2003. p. 13-47.

2. Sousa MC, Scatena JHG, Santos RV. O Sistema de Informação da Atenção à Saúde Indígena (SIASI): criação, estrutura e funcionamento. Cad Saúde Pública 2007; 23:853-61.

3. Leite MS. Transformação e persistência: antropologia da alimentação e nutrição em uma sociedade indígena amazônica. Rio de Janeiro: Editora Fiocruz; 2007.

4. Schuch I. Perfil socioeconômico e alimentar das famílias indígenas Kaingáng de Guarita - RS [Dissertação de Mestrado]. Campinas: Faculdade de Engenharia de Alimentos, Universidade Estadual de Campinas; 2001.

5. Menegolla IA, Fukuoka E, Rodrigues IH. Investigação de óbitos de crianças menores de 5 anos da Terra Indígena Guarita, Município de Redentora/ RS. Rev Bras Epidemiol 2002; 3 Suppl:229.

6. Menegolla IA, Drachler ML, Rodrigues IH, Schwingel LR, Scapinello E, Pedroso MB, et al. Estado nutricional e fatores associados à estatura de crianças da Terra Indígena Guarita, Sul do Brasil. Cad Saúde Pública 2006; 22:395-406.

7. Gilio J, Mioranza SL, Takizawa MGMH. Parasitismo intestinal em índios da reserva indígena de Rio das Cobras. Rev Bras Anál Clín 2006; 38:193-5.

8. Escobar AL, Santos RV, Coimbra Jr. CEA. Avaliação nutricional de crianças indígenas Pakaanóva (Warí), Rondônia, Brasil. Rev Bras Saúde Matern Infant 2003; 3:457-61.
9. Cardoso AM, Mattos IE, Koifman RJ. Prevalência de fatores de risco para doenças cardiovasculares na população Guarani-Mbyá do Estado do Rio de Janeiro. Cad Saúde Pública 2001; 17:345-54.

10. Lourenço AEP. Avaliação do estado nutricional em relação a aspectos socioeconômicos de adultos indígenas Suruí, Rondônia, Brasil [Dissertação de Mestrado]. Rio de Janeiro: Escola Nacional de Saúde Pública, Fundação Oswaldo Cruz; 2006.

11. Meyerfreund, D. Estudo da hipertensão arterial e de outros fatores de risco cardiovascular nas comunidades indígenas do Espírito Santo - BR [Tese de Doutorado]. Vitória: Programa de Pós-Graduação em Ciências Fisiológicas, Universidade Federal do Espírito Santo; 2006.

12. Mondini L, Canó EM, Fagundes U, Lima EES, Rodrigues D, Baruzzi RG. Condições de nutrição em crianças Kamaiurá - povo indígena do Alto Xingu, Brasil Central. Rev Bras Epidemiol 2007; 10:39-47.

13. Sampei MA, Cano EN, Fagundes U, Lima EES, Rodrigues D, Sigulem DM, et al. Avaliação antropométrica de adolescentes Kamayurá, povo indígena do Alto Xingu, Brasil Central (2000-2001). Cad Saúde Pública 2007; 23:1443-53.

14. Serafim MG. Hábitos alimentares e nível de hemoglobina em crianças indígenas Guarani, menores de 5 anos, dos Estados de São Paulo e do Rio de Janeiro [Dissertação de Mestrado]. São Paulo: Escola Paulista de Medicina; 1997.

15. Leite MS, Gugelmim SA, Santos RV, Coimbra Jr. CEA. Perfis de saúde indígena, tendências nacionais e contextos locais: reflexões a partir do caso Xavánte, Mato Grosso. In: Coimbra Jr. CEA, Santos RV, Escobar AL, organizadores. Epidemiologia e saúde dos povos indígenas no Brasil. Rio de Janeiro: Editora Fiocruz; 2003. p. 105-25. 
16. Morais MB, Alves GM, Fagundes Neto UL. Estado nutricional de crianças índias Terénas: evolução do peso e estatura e prevalência atual de anemia. J Pediatr 2005; 81:383-9.

17. Orellana JDY, Coimbra Jr. CEA, Lourenço AEP, Santos RV. Nutritional status and anemia in Suruí indian children, Brazilian Amazon. J Pediatr 2006; 85:383-8.

18. Ribas DLB, Sganzerla A, Zorzatto JR, Philippi ST. Nutrição e saúde infantil em uma comunidade indígena Teréna, Mato Grosso do Sul, Brasil. Cad Saúde Pública 2001; 17:323-31.

19. Fávaro T, Ribas DLB, Zorzatto JR, Segall-Corrêa AM, Panigassi G. Segurança alimentar em famílias indígenas Teréna, Mato Grosso do Sul, Brasil. Cad Saúde Pública 2007; 23:785-93.

20. Instituto Socioambiental. Povos indígenas no Brasil: línguas, quem, onde e quantos. http://www. socioambiental.org (acessado em 15/Jun/2007).

21. Zwetsch RE. Kaingáng, os limites do desenvolvimento. In: Leite AGO, organizador. Kaingáng, confrontação cultural e identidade étnica. Piracicaba: Editora Unimep; 1994. p. 15-58.

22. Hökerberg YHM, Duchiade MP, Barcellos C. Organização e qualidade da assistência à saúde dos índios Kaingáng do Rio Grande do Sul, Brasil. Cad Saúde Pública 2001; 17:261-72.

23. Diehl EE. Agravos na saúde Kaingáng (Terra Indígena Xapecó, Santa Catarina) e a estrutura dos serviços de atenção biomédica. Cad Saúde Pública 2001; 17:439-45.

24. World Health Organization. Physical status: the use and interpretation of anthropometry indicators of nutritional status. Geneva: World Health Organization; 1995. (Technical Report Series, 854).

25. World Health Organization. WHO child growth standards. http://www.who.int/childgrowth (acessado em 24/Fev/2007).

26. Hamill PVV, Drizd TA, Johnson CL, Reed RB, Roche AF, Moore WM. Physical growth: National Center for Health Statistics percentiles. Am J Clin Nutr 1979; 32:607-29.

27. Ribas DLB. Saúde e nutrição de crianças indígenas Teréna, Mato Grosso do Sul, Brasil [Tese de Doutorado]. São Paulo: Universidade de São Paulo, 2001.

28. Ribas DLB, Philippi ST. Aspectos alimentares e nutricionais de mães e crianças indígenas Teréna, Mato Grosso do Sul. In: Coimbra Jr. CEA, Santos RV, Escobar AL, organizadores. Epidemiologia e saúde dos povos indígenas no Brasil. Rio de Janeiro: Editora Fiocruz; 2003. p. 73-87.

29. Santos RV. Crescimento físico e estado nutricional de populações indígenas brasileiras. Cad Saúde Pública 1993; 9 Suppl 1:46-57.

30. Pesquisa Nacional sobre Demografia e Saúde - PNDS 1996. http://www.saude.gov.br/nutricao/ documentos/PesquisaNacDemografiaSaude.pdf (acessado em 05/Ago/2005).

31. Instituto Brasileiro de Geografia e Estatística. Pesquisa de Orçamentos Familiares. Antropometria e análise do estado nutricional de crianças e adolescentes no Brasil. Rio de Janeiro: Instituto Brasileiro de Geografia e Estatística; 2006.
32. Ministério da Saúde/Rede Integrada de Informações para a Saúde. Indicadores de morbidade e fator de risco: taxa de prevalência de déficit ponderal para a idade em crianças menores de cinco anos. http://www.saude.gov.br (acessado em 28/ Jan/2008).

33. Dufour DL. Diet and nutritional status of Amerindians: a review of the literature. Cad Saúde Pública 1991; 7:481-502.

34. Capelli JCS, Koifman S. Avaliação do estado nutricional da comunidade indígena Parkatêjê, Bom Jesus do Tocantins, Pará, Brasil. Cad Saúde Pública 2001; 17:433-7.

35. Morais MB, Fagundes Neto U, Mattos AP, Baruzzi RG. Estado nutricional de crianças índias do Alto Xingu em 1980 e 1992 e evolução pondero-estatural entre o primeiro e o quarto anos de vida. Cad Saúde Pública 2003; 19:543-50.

36. Lima RV. Avaliação do estado nutricional da população indígena da comunidade Terra Preta, Novo Airão, Amazonas [Dissertação de Mestrado]. Manaus: Universidade Federal do Amazonas/Fundação Oswaldo Cruz; 2004.

37. Gugelmin SA, Santos RV. Ecologia humana e antropometria nutricional de adultos Xavánte, Mato Grosso, Brasil. Cad Saúde Pública 2001; 17:313-22.

38. Santos RV, Coimbra Jr. CEA. Socioeconomic differentiation and body morphology in the Suruí of Southwestern Amazonia. Curr Anthropol 1996; 37:851-6.

39. Leite MS, Santos RV, Gugelmim SA, Coimbra Jr. CEA. Crescimento físico e perfil nutricional da população indígena Xavánte de Sangradouro-Volta Grande, Mato Grosso, Brasil. Cad Saúde Pública 2006; 22:265-76

40. Arruda HO, Filho JPBV, Ortiz V, Srougi M. PSA e medidas antropométricas em índios da Amazônia: avaliação da comunidade Parkatejê. Rev Saúde Pública 2003; 37:624-8.

41. Gimeno SGA, Rodrigues D, Pagliaro H, Cano EM, Lima EES, Baruzzi RG. Perfil metabólico e antropométrico de índios Aruák: Mehináku, Waurá e Yawalapití, Alto Xingu, Brasil Central, 2000/2002. Cad Saúde Pública 2007; 23:1946-54.

42. Romani SAM, Lira PIC. Fatores determinantes do crescimento infantil. Rev Bras Saúde Matern Infant 2004; 4:15-23.

43. Uchimura TT, Szarfarc SC, Latorre MRDO, Uchimura NS, Souza SB. Anemia e peso ao nascer. Rev Saúde Pública 2003; 37:397-403.

44. Ministério da Saúde/Rede Integrada de Informações para a Saúde. Indicadores de morbidade e fator de risco: proporção de nascidos vivos com baixo peso ao nascer. http://www.saude.gov.br (acessado em 28/Jan/2008).

45. Pícoli RP, Carandina L, Ribas DLB. Saúde maternoinfantil e nutrição de crianças Kaiowá e Guarani, Área Indígena de Caarapó, Mato Grosso do Sul, Brasil. Cad Saúde Pública 2006; 22:223-7.

46. Jelliffe DB. Nutrición infantil en países en desarrollo. Manual para trabajadores especializados. México DF: Limusa; 1976. 
47. Monteiro CA, Benicio MHA, Iunes RF, Gouveia NG, Cardoso MAA. Evolução da desnutrição infantil. In: Monteiro CA, organizador. Velhos e novos males da saúde no Brasil: a evolução do país e de suas doenças. São Paulo: Editora Hucitec/Núcleo de Pesquisas Epidemiológicas em Nutrição e Saúde, Universidade de São Paulo; 1993. p. 93-114.

48. Olinto MTA, Victoria CG, Barros FC, Tomasi E. Determinantes da desnutrição infantil em uma população de baixa renda: um modelo de análise hierarquizado. Cad Saúde Pública 1993; 9 Suppl 1:14-27.

49. Post CLA, Victora CG, Barros AJD. Entendendo a baixa prevalência de déficit de peso para estatura em crianças brasileiras de baixo nível sócio-econômico: correlação entre índices antropométricos. Cad Saúde Pública 2000; 16:73-82.
50. Ferreira KR. Importância do baixo peso ao nascer sobre a prevalência da desnutrição infantil em Ponte do Pasmado, Itinga - MG. Revista de Iniciação Cientifica Newton Paiva 1996; 12 Suppl 1:43-8.

51. Engstrom EM, Anjos LA. Déficit estatural nas crianças brasileiras: relação com condições sócio-ambientais e estado nutricional materno. Cad Saúde Pública 1999; 15:559-67.

52. Guimarães LV, Latorre MRDO, Barros MBA. Fatores de risco para a ocorrência de déficit estatural em pré-escolares. Cad Saúde Pública 1999; 15:605-15.

Recebido em 13/Fev/2008

Versão final reapresentada em 08/Jul/2008

Aprovado em 21/Jul/2008 